\title{
L'eau comme élément symbolique dans La Chartreuse de Parme de Stendhal, dans Les Années de Virginia Woolf et dans Le Guépard de Tomasi di Lampedusa
}

\author{
Maruggi, Maria \\ Université de Strasbourg - L'Europe des Lettres EA 1337, maria.maruggi@alice.it
}

\begin{abstract}
Resumen
En La Cartuja de Parma de Stendhal, Los Años de Virginia Woolf y en El Guepardo de Tomasi di Lampedusa, el agua está presente como elemento simbólico y se carga de sentidos profundos. En La Cartuja de Parma el agua remite a la nostalgia de la patria idealizada, Italia. En particular el recuerdo de la vista del lago de Como, antes de que el escritor abandonara Italia en 1821, aparece recurrentemente en el texto. Este lago es «sublime» a los ojos de Fabricio, para quien «nada tan bello se puede ver en el mundo ». Como muestra Gaston Bachelard, el agua puede asumir un doble sentido, maternal y de ensueño. La obra de Stendhal es la perfecta ilustración de ello. En Los Años, los personajes, mirando el agua, parecen revivir las mismas experiencias interiores que la escritora había vivido y descrito en su diario íntimo. Del agua emergen las imágenes del pasado de Rosa. Se trata de un pasado enterrado aún doloroso. De esa manera el agua fascina a los personajes que se paran a contemplar, como hipnotizados, este elemento natural. En El Guepardo, el agua, dotada de gran valor simbólico, también está presente a nivel estilístico. Cuando el príncipe de Salina muere en su habitación del hotel situada al borde del mar, su muerte es descrita con imágenes acuáticas: «ya no era un río que se desplegaba de él, sino un océano, una tempestad, erizada de espuma y grandes olas desatadas [...] ». En esta lectura comparada veremos como Stendhal, Tomasi di Lampedusa y Virginia Woolf, han representado en sus novelas la nostalgia, la profundidad del alma y el binomio vida-muerte a través del agua.
\end{abstract}

Palabras clave : agua ; nostalgia ; vida ; muerte ; mito.

\section{Résumé}

Dans La Chartreuse de Parme de Stendhal, Les Années de Virginia Woolf et dans Le Guépard de Tomasi di Lampedusa, l'eau est présente en tant qu'élément symbolique et se charge de significations profondes. Dans La Chartreuse de Parme l'eau renvoie à la nostalgie de la patrie idéalisée, l'Italie. En particulier le souvenir de la vue du lac de Côme, avant que l'écrivain quitte l'Italie en 1821, revient dans le texte à plusieurs reprises. Ce lac est «sublime » aux yeux de Fabrice, pour qui «rien d'aussi beau ne peut se voir au monde ». Comme l'a montré Gaston Bachelard, l'eau, peut assumer un double caractère, maternel et de rêverie. L'œuvre de Stendhal en est la parfaite illustration. Dans Les Années, les personnages, en regardant l'eau, semblent revivre les mêmes expériences intérieures que l'écrivaine avait vécues et décrites dans son journal intime. De l'eau émergent les images du passé de Rose. Il s'agit d'un passé enseveli mais toujours douloureux. Ainsi, l'eau fascine les personnages qui s'arrêtent et contemplent, comme hypnotisés, cet élément naturel. Dans Le Guépard, l'eau se charge de fonctions symboliques mais elle est ainsi présente au niveau du style. Lorsque le prince Salina meurt dans sa chambre d'hôtel située au bord de la mer, la description intérieure de sa mort se fait par des images aquatiques : "Ce n'était plus un fleuve qui déferlait de lui, mais un océan, en tempête, hérissé d'écume et de grosses vagues déchaînées [...]». À travers une lecture comparée, nous verrons comment Stendhal, Tomasi di Lampedusa et Virginia Woolf, ont représenté dans leurs romans la nostalgie, la profondeur de l'âme ainsi que le binôme vie-mort à travers l'eau.

Mots-clés : eau ; nostalgie; vie ; mort ; mythe. 


\begin{abstract}
In the novels The Chartehouse of Parme by Stendhal, The Years by Virginia Woolf and The Leopard by Tomasi di Lampedusa, water is defined as a symbolic element imbued with profound meanings. In The Chartehouse of Parme water refers to the nostalgia of Italy an idealized country. In particular the memory of Lake Como's view, when the writer leaves Italy, is often mentioned in the novel. This lake is «sublime» to Fabrice's eyes for whom «nothing so beautiful can be seen in the world». As demonstrated by Gaston Bachalard, in the work of Standhal water has two different natures, the maternal one and the reverie one. In The Years by looking the water the characters seem to experience again the same feelings which the writers had in life and described in her private diary. Rosa's images of the past emerge from the contemplation of water. This long-buried past is still painful to her. In the same way all the other characters are enchanted and hyptonized coming across the sight of this natural element. In The Leopard water is embodied in symbolic functions and in the level of style. As Prince Salina is dying in a hotel room on the seashore his inward death is depicted with aquatic images «Now it was not a river erupting over him but an ocean, tempestuous, all foam and raging white-flecked waves [...] ». Through this comparative reading of Stendhal, Virginia Woolf and Tomasi di Lampedusa it will be demonstrated how nostalgia, the detph of soul, life and death are depicted with the image of water.
\end{abstract}

Keywords : water ; nostalgia ; life ; dead ; myth.

\title{
Introduction
}

Dans La Chartreuse de Parme de Stendhal, Les Années de Virginia Woolf et dans Le Guépard de Tomasi di Lampedusa, l'eau est un élément symbolique dont la contemplation renvoie au concept de sublime chez Stendhal, et elle fait ressurgir des images violentes et apaisantes chez les personnages de Woolf. L'eau, en outre, devient un élément qui s'associe au binôme vie-mort chez l'écrivaine anglaise et dans Le Guépard de Lampedusa.

Notre étude se propose d'établir une lecture comparative entre ces trois écrivains, en analysant aussi les différences qui marquent chez eux le traitement de l'élément aquatique. Il est important de souligner l'amour inconditionnel que Lampedusa portait pour les œuvres de Stendhal et Virginia Woolf, maîtres de cette temporalité subjective également présente dans son roman.

Dans la Chartreuse, l'eau est un élément participant du sublime. Stendhal détourne un peu le terme « sublime » de son sens originel. Pour l'écrivain, le sublime est à la fois un idéal auquel les personnages doivent aspirer, une manière de ressentir les beautés de l'univers, mais il est aussi, selon l'acception classique, ce qu'il y a de plus beau dans la nature. Selon la tradition le sublime a partie liée à la nature, Kant avait souligné comment la nature et ses phénomènes suscitent dans celui qui les contemple l'idée de son infinité. Nous analyserons comment la Chartreuse nous présente un sublime de la nature à travers l'eau.

Dans la Chartreuse, l'eau accompagne le parcours de maturation du héros, sert ainsi de cadre à ses évolutions. Nous montrerons comment la contemplation de l'eau reflète les sentiments du héros. Ainsi, nous donnerons à voir comment cet élément naturel sert de cadre à la vie et aux aventures de Fabrice. Cette étude nous permettra ainsi de nous questionner sur une signification plus profonde de l'eau dans le roman de Stendhal. En effet, cet élément renvoie également à la nostalgie d'une patrie idéalisée par Stendhal, l'Italie. Peut-on affirmer que chez Stendhal la rêverie de l'eau se rapporte à l'image de la mère ? Stendhal, en se réinventant une patrie, essaie-t-il de retrouver les joies de l'enfance perdues après la mort de la mère aimée ?

De manière plus contemporaine, l'eau constitue un élément des poétiques respectives de Woolf et de Lampedusa. Ainsi, nous démontrerons comment dans Les Années, la contemplation de l'eau cache des images refoulées chez l'écrivaine : des images trop douloureuses chez les protagonistes qui regardent l'eau. En outre, cet élément offre un refuge contre les agressions de la société, alors que dans Le Guépard les images aquatiques deviennent des métaphores du travail intérieur du corps agonisant du Prince Salina. 


\section{L'eau sublime et nostalgique dans La Chartreuse de Parme de Stendhal}

Dans La Chartreuse de Parme, le lac de Côme et sa contemplation accompagnent le chemin existentiel et la maturation de Fabrice qui, de personnage naïf et peu héroïque de la première partie du roman, deviendra un vrai héros dans sa prison d'amour dans la deuxième partie. C'est en contemplant le lac, que Fabrice montre son penchant pour le sublime, il est capable de s'adonner à des sentiments d'exception que seuls les êtres singuliers, selon Stendhal, peuvent éprouver à la vue du beau. Si Fabrice est tout sauf un héros alors qu'il prend part à la bataille de Waterloo, il en en va tout autrement lorsqu'il contemple les eaux. Cette méditation de l'eau a le caractère de ce que Kant nomme le sublime noble, un sublime qui dispose l'âme à la rêverie mélancolique. Le lac sert de cadre à la rencontre de Fabrice et de Clélia, la jeune fille pour laquelle Fabrice va accepter avec joie la vie en prison.

Lors de cette rencontre, Fabrice est en train de fuir après avoir été dénoncé par son frère Ascagno comme espion à la solde de Napoléon. Fabrice, qui se demandait encore si «ce qu'il avait vu, était-ce une bataille » et si «cette bataille était-elle Waterloo ? » (Stendhal, 1998 : 95), part pour Milan aidé par de sa tante Gina, la comtesse Pietranera, et de sa mère, la marquise Del Dongo. Leur voiture est arrêtée aux portes de Milan, où les gendarmes ont également arrêté la voiture du général Fabio Conti et sa fille Clélia. C’est près du lac que Fabrice, épris de la jeune fille, pense, sans savoir qu'il il y aura une part de vérité dans ses pensées : «Ce serait une charmante compagne de prison » (Stendhal, 1998 : 101). En effet, dans la suite du récit, quand Fabrice sera conduit en prison, Clélia, l'apercevant dehors conduit par les gardes, se souviendra de leur rencontre près du lac : «Qui m’eut dit, pensa-t-elle, que je le reverrai pour la première fois dans cette triste situation, quand je le rencontrai sur la route du lac de Côme ? " (Stendhal, 1998 : 285). Au même instant, Fabrice pense en la regardant : «Comme elle est embellie depuis notre rencontre près de Côme ! " (Stendhal, 1998 : 287). De même, dans la prison, Fabrice s'étonne du bonheur qu'il ressent, il est heureux, et l'eau, la vue du Pô cette fois, sert de nouveau de cadre à son bonheur amoureux.

L'eau, à la fin du roman, adoucira le sort du héros, alors que Clélia sera morte et qu'il se retirera volontairement dans la Chartreuse de Parme, « située dans les bois voisins du Pô, à deux lieux de Sacca » (Stendhal, 1998 : 534). Le paysage aquatique constitue l'ultime refuge.

Cependant, c'est aux chapitres huit et neuf de la première partie du roman que la contemplation de l'eau s'accomplit au sens le plus esthétique et le plus sublime du terme. Après un mois à la cour de Parme, Fabrice décide de tromper l'ennui en séduisant l'actrice Marietta, dont Giletti revendique la possession. Cependant, il ne peut pas profiter de Marietta parce que sa mère, sous le conseil de Gina (qui est jalouse de cette actrice), demande à Fabrice d'aller lui rendre visite. Fabrice part pour Belgirate et après avoir rencontré sa mère décide de rendre visite à l'abbé Blanès. En chemin, notre héros, s'arrête sur les rives du lac de Côme : "Il était minuit, et Fabrice pouvait espérer de ne rencontrer aucun gendarme. [...] Les eaux et le ciel étaient d'une tranquillité profonde ; l'âme de Fabrice ne put résister à cette beauté sublime; il s'arrêta, puis s'assit sur un rocher qui s'avançait dans le lac, formant comme un petit promontoire » (Stendhal, 1998 : 173).

Stendhal justifie l'attitude romantique de son héros en précisant que,

Fabrice avait un cœur italien ; j’en demande pardon pour lui : ce défaut, qui le rendra moins aimable, consistait en ceci : il n'avait de vanité que par accès, et l'aspect seul de la beauté sublime le portait à l'attendrissement, et ôtait à ses chagrins leur pointe âpre et dure. Assis sur son rocher isolé, n’ayant plus à se tenir en garde contre les agents de police, protégé par la nuit profonde et le vaste silence, de douces larmes mouillèrent ses yeux, et il trouva là, à peu de frais, les moments les plus heureux qu’il eut goûtés depuis longtemps (Stendhal, 1998 : 173).

Après les révélations de l'abbé Blanès quant à la prison qui l'attend, une prison plus dure que celle de Waterloo, Fabrice retourne sur ces rives et pense que,

Puisqu'il semble que je ne dois pas connaître l'amour, ce seront toujours là pour moi les grandes sources de félicité ; je voudrais, avant de mourir, aller revoir le champ de bataille de Waterloo [...] Ce pèlerinage accompli, je reviendrais 
souvent sur ce lac sublime ; rien d'aussi beau ne peut se voir au monde, du moins pour mon cœur. À quoi bon aller si loin chercher le bonheur, il est là sous mes yeux ! (Stendhal, $1998: 185$ )

Ces passages sont décisifs pour la compréhension, d'une part du rapport sublime de Fabrice à l'eau ; d'autre part, de l'amour de Stendhal pour l'Italie. C'est l'écrivain lui-même qui justifie l'excès de sentiments que Fabrice éprouve en regardent les eaux : en attribuant à Fabrice une réaction excessive devant la beauté du lac (réaction liée à ses origines italiennes), Stendhal fait de son héros un être rare qui, grâce à sa pureté, peut jouir de la contemplation du beau. Fabrice peut vivre les moments les plus heureux en regardant ce lac et, comme le souligne Stendhal, cela se fait à peu de frais. Comme l'a constaté Dominique Fernandez dans le Dictionnaire amoureux de Stendhal : "Aux hommes "à argent", Stendhal oppose les hommes "à imagination". Aux âmes "froides” celles qui sont disposées "à sentir” " (Fernandez, 2013 : 55). La contemplation de la beauté du lac est de pure gratuité. Rien n’est plus beau au monde que ce lac aux yeux de Fabrice. La rêverie mélancolique à laquelle il s'abandonne lui fait oublier le péril qu'il court en restant dans les territoires autrichiens. À travers l'expérience esthétique de son héros, Stendhal montre son amour pour un paysage et pour une patrie qu'il a dû abandonner en 1821, à cause de ses idées anti-autrichiennes. Avant de quitter la patrie qu'il s'était inventée, Stendhal retourna visiter le lac de Côme. Comme son héros, Stendhal a connu la fuite. Comme l'a observé Gaston Bachelard dans L'eau et les rêves, « Le pays natal est moins une étendue qu'une matière ; c'est un granit ou une terre, un vent ou une sécheresse, une eau ou une lumière. C’est en lui que nous matérialisons nos rêveries ; c'est par lui que notre rêve prend sa juste substance ; c'est à lui que nous demandons notre couleur fondamentale » (Bachelard, 1942 : 11-12).

Stendhal aurait fait de l'Italie sa véritable patrie ; les rêveries mélancoliques auxquelles s'abandonne son héros seraient le reflet de sa propre nostalgie. La contemplation esthétique apaise, chez Fabrice comme chez son auteur, le sentiment du malheur. Les deux sont en fuite, les deux sont tourmentés par le pouvoir autrichien, les deux ont vécu leur amour pour Napoléon, tous deux rêvent en contemplant l'eau.

Si Fabrice passe son enfance près du lac de Côme et s'il veut y retourner avant de mourir, nous pouvons nous demander si ce n'est pas une projection de Stendhal lui-même, et si chez lui l'eau ne renvoie pas à l'image de la mère mourante. Comme le dit Bachelard, l'eau serait une projection de la mère.

Dans la Chartreuse ainsi que dans Les Années de Virginia Woolf, la contemplation de l'eau est chargée de significations profondes.

\section{L’eau dans Les Années ou les images refoulées chez Virginia Woolf}

Dans Les Années, Virginia Woolf décrit l'histoire de la famille Pargiter de 1880 au Temps présent, les années 1930. Dans ce roman, les ruptures temporelles et spatiales suivent les réflexions des protagonistes sur des thèmes tels que l'altérité de leur propre être, le rôle de la femme dans la société, la justesse de la guerre, la mort et la nostalgie de la jeunesse, les interrogations sur le sort du genre humain, sur la possibilité qu'il devienne meilleur.

Dans Les Années, tout comme dans Les Vagues, nous ne parvenons pas à délimiter des personnages secondaires au sens strict du terme : chacun est porteur d'une vérité profonde. Dans ce roman, la contemplation de l'eau est liée à un traitement particulier du temps narratif. En effet, dans Les Années, le passé et les souvenirs se mêlent sans cesse au présent.

De plus, il est important de souligner que certaines scènes décrites dans le roman ont eu un fort impact émotif chez l'écrivaine au moment de les relater. Woolf elle-même dans son Journal (Vendredi, 6 septembre 1935) parle des crises entraînées par la description de la Serpentine vue par Maggie et sa mère : « Maggie regarda dans la direction indiquée par sa mère. Il y avait la Serpentine, rouge dans le soleil couchant ; les arbres regroupés, sculptés, perdant leur détails ; et l'architecture spectrale du petit pont, blanc à l'extrémité, composant la scène. Les lumières - la lumière du soleil et la lumière artificielle - étaient étrangement mêlées » (Woolf, 2012 : 828).

Il s'agit d'une scène floue qui respecte probablement la réserve de l'écrivaine qui initialement ne voulait pas inclure ce passage. Le paysage perd de sa consistance, et les lumières qui se confondent sont là pour augmenter l'impression de fluidité. 
La contemplation de l'eau révèle ainsi les vieux souvenirs de Rose Pargiter. C’est le printemps des années 1910, Rose a quarante ans et elle est en ces années une suffragette active. Elle va rendre visite à ses cousines pauvres, Maggie et Sara et durant le trajet,

\begin{abstract}
Elle s'arrêta dans l'une des petites alcôves qui étaient creusées sur le pont, par habitude. Les gens s'arrêtaient toujours pour voir la Tamise. Elle coulait vite, d'une couleur or boueux ce matin, avec des larges surfaces lisses et des rides, car la marée était haute. Et il y avait l'habituel remorqueur et les habituelles péniches et leurs bâches noires, laissant apercevoir du blé. L'eau tourbillonnait autour des arches. Alors qu'elle était là à regarder l'eau, un sentiment enfoui commença à dessiner un motif sur la surface du fleuve. Ce motif était douloureux. Elle se souvint de s'être trouvée là à pleurer un certain soir de fiançailles ; ses larmes avaient coulé, et il lui semblait que son bonheur s'était lui aussi écoulé (Woolf, 2012 : 854).
\end{abstract}

À la lecture de cette description, on pourrait penser que le temps s'est arrêté. On y retrouve les remorqueurs et les gabarres de toujours, lieu du souvenir et lieu actuel se situent au même endroit. Le rythme rapide du cours de l'eau, la violence des tourbillons anticipent la violence des images qui ressurgiront à la vue de l'eau. Rose se souvient d'avoir pleuré sur le même pont, le passé revient avec toute sa violence et sa douleur. C'est l'eau qui dessine et ressuscite cette image douloureuse appartenant au passé. L’immobilité du temps est rendue par l’image du panorama, présenté dans son éternité tandis que la félicité de Rose a été emportée en l'espace d’une nuit.

Comme l'a souligné Marie-Paule Vigne dans Le thème de l'eau dans l'œuvre de Virginia Woolf, «L'eau de Virginia Woolf est une eau vue de loin, une eau vue de haut » (Vigne, 1984 : 389). De cette position aérienne, l'eau dessine les images d'un passé révolu.

La contemplation de l'eau dans Les Années ne ressuscite pas seulement le passé douloureux, tout comme dans la Chartreuse elle devient aussi un refuge à l'abri du monde et de ses règles. C’est le cas pour Kitty, le personnage qui a toujours mis de côté ses vrais sentiments afin de respecter les règles que la position aristocratique de sa famille lui a imposées ; elle peut vivre sa rébellion à l'égard des normes. Après le bal qu'elle a donné chez elle, Kitty prend le train de nuit et se laisse emporter par la solitude et ses souvenirs. Arrivée dans sa maison de campagne elle se promène et descend jusqu’à la rivière :

L'eau la fascinait toujours. La rivière du Nord, avec son courant rapide, descendait la lande ; elle n'était jamais lisse et verte, jamais profonde et paisible comme les rivières du Sud. Elle bondissait ; elle se précipitait. Elle s'évasait, rouge, jaune et brun transparent, sur les cailloux de son lit. Appuyant ses coudes sur la balustrade, elle la regarda faire jaillir des diamants et des traits de flèches acérés en passant sur les pierres. Elle écouta. Elle connaissait les sons différents que faisait résonner la rivière en été et en hiver ; à présent elle se hâtait, elle s’emballait (Woolf, 2012 : 952 ).

Nous ne pouvons nous empêcher de penser à la fascination que l'eau a toujours exercé sur Woolf. Kitty nous rappelle l'image de l'écrivaine fascinée par cet élément naturel qui sera par la suite l'élément qui accueillera son corps au moment de sa mort. Dans ce passage, Kitty montre une connaissance profonde de la rivière. Comme le précise Bachelard dans la Poétique de la rêverie, l’on ne peut rêver que de ce que l'on connaît bien. L'eau, l'élément de la rêverie, nous apporte un bonheur infini parce qu'elle donne un libre cours à l'imagination et permet l'épanouissement de l'être.

\title{
2.1. Virginia Woolf ou la mort par l'eau
}

Tout comme Kitty, la protagoniste des Années, Virginia Woolf connaît l'élément aquatique : jusqu’à la mort de sa mère elle passait tous les étés à St Ives, la baie où son père, Leslie Stephen, avait acheté Talland House en 1881. Une fois mariée avec Leonard, l'écrivaine fera encore des voyages en Cornouailles. Dans sa résidence à Monk’s House, Virginia Woolf aimait à s'asseoir près du bassin ou se promener sur l'Ouse. L'eau, élément omniprésent dans l'œuvre de Virginia Woolf, sera l'élément qui accueillera son corps dans la mort ; la critique n’a pu s'empêcher, tout comme nous 
lecteurs, de faire émerger cette étrange coïncidence entre l’œuvre et la mort de l'écrivaine, toutes les deux imprégnées de la symbolique de l'eau.

Une approche psychanalytique pourrait nous faire penser que l'eau, comme nous l'avons proposé pour Stendhal, se confond avec l’image de la mère : «Quoi d'étonnant » précise Marie-Paule Vigne, "si elle devient rêve et nostalgie chez celles qui, telle Virginia Woolf, ressentent intensément en leur corps et en leur esprit l'arrachement, la division, l’aliénation ? La mer est plus qu'un havre, elle est l'unité perdue et retrouvée, la totalité reformée et refermée » (Vigne, 1984 : 548).

Nous avons souligné comment Lampedusa s’inspire de la poétique de Stendhal et de Virginia Woolf pour son œuvre, notamment pour ce qui est du traitement du temps dans la narration. Ainsi, lorsque Lampedusa consacre un chapitre à Woolf dans ses leçons de Letteratura inglese, il utilise les métaphores aquatiques pour rendre avec exactitude l'opération que l'écrivaine fait dans le traitement du temps (Lampedusa, 2004). Nous verrons comment, dans Le Guépard, l'écrivain pratique une intériorisation de l’élément aquatique.

\section{L’eau : image de la vie, de la mort dans Le Guépard de Tomasi di Lampedusa}

Dans Le Guépard, Lampedusa décrit la fin d'un monde, celui de l'aristocratie sicilienne, filtrée à travers la perspective de Don Fabrizio Salina. Tout comme chez Stendhal et Woolf, le passage du temps dans le roman de Lampedusa, est déterminé par le point de vue du héros. Ainsi, la mort de Don Fabrizio se déroule-t-elle de l’intérieur ; notre prince se voit mourir. L'eau sert de cadre à sa mort. Il agonise dans l’hôtel Trinacria à Palerme près de la mer : « Assis dans un fauteuil, ses très longues jambes enveloppées dans une couverture, sur le balcon de l'hôtel Trinacria, il sentait que la vie sortait de lui par longues vagues pressantes, avec un fracas spirituel comparable à celui de la cascade du Rhin. C’était à midi, un lundi de la fin de juillet, et la mer compacte, huileuse, inerte, s’étendait en face de lui » (Lampedusa, 2007 : 257).

La mer immobile à Palerme répond symétriquement à l'eau intérieure, aux vagues et au fracas spirituel de Don Fabrizio qui compare le cours de sa mort aux mouvements impétueux des eaux. En effet, l'eau est également l'élément par lequel Lampedusa métaphorise l'agonie du prince. Cette agonie recouvre un chapitre entier et les images aquatiques accompagnent ce voyage vers la mort. Au moment où Don Fabrizio se contemple dans le miroir et ne reconnaît plus son visage, parce que « cela arrive à tout le monde : on meurt avec un masque sur le visage » (Lampedusa, 2007 : 270), le prince pense que la même chose arrive aux jeunes qui meurent. « Et si en lui, qui était vieux, le fracas de la vie en fuite était si puissant, quel avait dû être le tumulte des réservoirs encore pleins de ces pauvres jeunes corps qui se vidaient en un instant? » (Lampedusa, $2007: 260-261)$.

L'eau devient alors l'image de la vie. Elle représente la puissance vitale mais elle est aussi, aux yeux de Don Fabrizio une puissance ténébreuse. Tandis que le silence du dehors contrastait avec le grondement intérieur et que ressurgissent les souvenirs des êtres chers, face au paysage ensoleillé qui l'empêchait de penser à sa maison, qu' il voyait alors comme en rêve et qui lui semblait étrangère, alors qu’ il lui semblait « qu’il n’avait plus à présent que ce corps épuisé » qu'il aperçut « cette chute d'eaux ténébreuses vers l'abîme » et qu' il était ce «naufragé à la dérive sur un radeau, en proie aux courants indomptables » (Lampedusa, 2007 : 262). L’eau, de réservoir vital devient symbole de mort, devient un abîme et laisse le mourant naufragé en proie à sa violence.

Le prince voit défiler les moments les plus heureux de sa vie, et le sentiment de tradition et de pérennité s’expriment, dans le texte, dans l’eau, dans « le temps glacé » (Lampedusa, 2007 : 266).

Les souvenirs des meilleurs moments de sa vie prennent un soudain relief, ces moments vécus qui se résument à deux ou trois années de vie au plus. À ces souvenirs se mêlent les images des femmes rencontrées dans sa vie. De celle, par exemple, qu'il a entrevue la veille à la gare de Catane en tenue de voyage et qui avait cherché son visage, il se souvient des « sourires menteurs » de ses proches qui l'attendaient à la gare, et du «déferlement soudain des cataractes [...]» (Lampedusa, 2007 : 267).

L’image aquatique est vécue à la fois en souvenir et comme accompagnement de l'instant, le déferlement des cascades laisse à penser que le prince parcourt son voyage vers la mort à travers les sensations associées à l'eau, les métaphores aquatiques illustrant tout autant sa vie que sa mort prochaine. 
Ces images, il les aperçoit en mourant, et l'expérience du mourir est décrite par analogie à la tempête suivie du calme d'après la mort. « Ce n’était plus un fleuve qui déferlait de lui, mais un océan, en tempête, hérissé d’écume et de grosses vagues déchaînées [...] » (Lampedusa, 2007 : 268). Ainsi, de simple fleuve, ce courant vital qui déferle de lui devient un océan menaçant à cause de la tempête et des vagues violentes. La vie avec toute sa violence s'en va, pour laisser place à la pénétration de la mort. La femme en costume de voyage qu'il avait vue à la gare se fraie un chemin parmi les proches qui entourent le mourant :

C’était elle, la créature désirée depuis toujours qui venait le chercher : c’était étrange qu'elle, si jeune, lui eut cédé ; l'heure du départ du train devait approcher. Arrivée face à lui, elle souleva sa voilette et ainsi, pudique mais prête à être possédée, elle lui apparut plus belle qu’il ne l'avait jamais vue dans les espaces stellaires. Le fracas de la mer se calma tout à fait (Lampedusa, $2007: 268$ ).

Cette mort qui coïncide avec la possession de cette femme et avec le calme de la mer est emblématique du sens aquatique de la mort pour le prince. En effet, comme l’ont souligné Giorgio Bassani dans sa préface aux nouvelles de Lampedusa, et Salvatore Silvano Nigro dans le chapitre de Il principe fulvo, intitulé « Il mare, la morte, l'immortalità », la nouvelle Le professeur et la Sirène, que l'écrivain écrit après Le Guépard, est indissociable de ce roman si l'on veut comprendre le lien indissociable que l'eau et la vie d'une part, l'eau et la mort, de l'autre, entretiennent dans l'imaginaire de Lampedusa.

L’image mythique de la sirène sensuelle et immortelle qui émerge de l'eau, donne un sens profond à la représentation de la mort chez le prince Salina. Dans cette nouvelle, le vieux professeur La Ciura, vit dans le souvenir de jeunesse de l'union passionnelle et érotique avec la sirène Lighea, alors qu'il se préparait à passer l'examen pour la chaire de grec.

Lighea symbolise l'amour suprême et sensuel lié au charme mythique des sirènes. Cette créature de la mer a fait connaître un amour pur à La Ciura, un sentiment qui n'a rien de l'amour égoïste des relations humaines. Ce vieux professeur à la fin du récit décide de rejoindre sa jeune amante dans les profondeurs de la mer. Comme Don Fabrizio, La Ciura vit un amour passionnel et érotique dans le temps de la mort, associée à l'image d’une jeune femme en voyage ou d'une sirène qui habite les profondeurs de la mer.

\section{Conclusion}

Nous avons démontré comment l'eau devient chez Stendhal, Virginia Woolf et Tomasi di Lampedusa, un symbole non seulement poétique, mais aussi existentiel. L’eau se charge chez nos écrivains de plusieurs significations : d’élément du sublime à équivalent psychique de projection maternelle, l'élément aquatique devient également le symbole de la vie, de la mort, et permet le retour au mythe grec.

L'eau est un élément important dans la vie de Fabrice, et sa vue lui inspire des sentiments qui relèvent du sublime. Chez Stendhal et chez Woolf, la contemplation de l'eau devient aussi un refuge à l'abri de la violence de l'histoire pour Fabrice et des règles de la société pour Kitty.

Chez Virginia Woolf et chez Lampedusa, l'eau devient une métaphore symbolique de vie et de mort. La mort par l'eau est la mort de Virginia Woolf. L'eau, dans ses romans, n'est pas seulement quelque chose que l'on contemple, mais elle est la substance même de sa poétique. Ainsi, l’eau est intériorisée dans Le Guépard, ce n’est plus seulement un élément du paysage extérieur qui reflète les états d’âme du héros et qui fait cadre à ses joies et à ses malheurs. L'eau est un élément spirituel, sa violence et ses mouvements deviennent les métaphores de la vie et de la mort du prince Salina. De l'eau émerge ainsi la sirène du mythe classique. La pénétration de la mort, de l'étoile Venus métamorphosée en femme sensuelle dans Le Guépard, la mort dans l'eau du professeur La Ciura qui rejoint la sirène autrefois possédée, dans Le professeur et la sirène, donnent un sens nouveau à la mort. Toutefois, cette image de la féminité, requiert une autre lecture commune aux trois œuvres et qui renvoie à une signification plus profonde. En effet, l'eau, comme élément symbolique, et sans doute en premier lieu, doit être comprise comme la résurgence de la figure maternelle. 


\section{Références bibliographiques}

BACHELARD, Gaston (1942). L'eau et les rêves, essai sur l'imagination de la matière. Paris : José Corti.

BACHELARD, Gaston (1960). La poétique de la rêverie. Paris : PUF.

BASSANI, Giorgio (1958). Prefazione a G. Tomasi di Lampedusa. I racconti. Milano : Feltrinelli.

FERnANDEZ, Dominique (2012). Dictionnaire amoureux de Stendhal. Paris: Plon Grasset.

Tomasi di LAmpedusA, Giuseppe (1958). Il Gattopardo. Milano : Feltrinelli. Le Guépard. Trad. de l’italien par Jean-Paul Manganaro (2007), postface de G. Lanza Tomasi. Paris : Seuil, coll. « Points-Grands romans ».

Tomasi di Lampedusa, Giuseppe (1961). La Sirena, in Racconti, pref. di Giorgio Bassani, Racconti. Milano : Feltrinelli. Le Professeur et la sirène. Trad. de l’italien par L. Bonalumi (2007). Préface de Giorgio Bassani. Paris : Seuil, coll. « Points ».

Tomasi di LAmpedusA, Giuseppe (1995). Opere. Introduzione e premesse di Gioacchino Lanza Tomasi, I racconti, Letteratura inglese, Letteratura francese a cura di Nicoletta Polo. Milano : I Meridiani Mondadori.

NigRo, Salvatore Silvano (2012). « Il mare, la morte, l’immortalità », dans Il Principe fulvo. Palermo : Sellerio. 45-78.

Stendhal (1841). La Chartreuse de Parme. Paris : Dupont. La Chartreuse de Parme. Préface et commentaire de Pierre-Louis Rey (1998). Paris : Pocket Classiques.

Vigne, Marie-Paule (1984). Le thème de l'eau dans l'œuvre de Virginia Woolf. Thèse doctorale. Bordeaux : Presses Universitaires de Bordeaux.

Woolf, Leonard (1953). A Writer's Diary. Virginia Woolf. London : Hogart-Press. Virginia Woolf. Journal d'un écrivain. Traduit de l’anglais par Germaine Beaumont (1958). Monaco : Éditions du Rocher.

Woolf, Virginia [1937] (2012). The Years. London : Hogarth Press. Les Années in Euvres romanesques, II. Éd. Paris : Gallimard, coll. « Bibliothèque La Pléiade ». 